\title{
ON THE SIGNATURES OF ORDERED SYSTEM LIFETIMES
}

\author{
N. BALAKRISHNAN *** AND \\ WILLIAM VOLTERMAN, ${ }^{* * * *}$ McMaster University
}

\begin{abstract}
The idea of the system signature is extended here to the case of ordered system lifetimes arising from a test of coherent systems with a signature. An expression is given for the computation of the ordered system signatures in terms of the usual system signature for system lifetimes. Some properties of the ordered system signatures are then established. Closed-form expressions for the ordered system signatures are obtained in some special cases, and some illustrative examples are presented.
\end{abstract}

Keywords: Coherent system; order statistics; system signature

2010 Mathematics Subject Classification: Primary 60K10

Secondary 90B25; 62G30

\section{Introduction}

In studying reliability systems, coherent systems arise naturally in many cases. Coherent systems are those whose reliability improves when one or more components are improved and for which each component is relevant to the lifetime of the system. In the study of such coherent systems, one measure that has become quite useful is the system signature introduced by Samaniego [6] for coherent systems based on independent and identically distributed (i.i.d.) components with a continuous lifetime distribution. Some well-known examples of coherent systems are the series system, parallel system, and $k$-out-of- $m$ system, which is operational when at least $k$ of the $m$ components are functioning.

For a coherent system with $m$ components, the system signature is a vector of length $m, s=$ $\left(s_{1}, \ldots, s_{m}\right)$, with the $j$ th component representing the probability of the system failing due to the $j$ th ordered component failure. More formally, if the component lifetimes $X_{1}, \ldots, X_{m} \sim F$, where $F$ is continuous, and $X_{1: m}, \ldots, X_{m: m}$ represent their order statistics, then, if $T$ represents the lifetime of the system, for $j=1,2, \ldots, m$, we have

$$
s_{j}=\mathbb{P}\left(T=X_{j: m}\right)
$$

As noted in [6], the above representation is free of $F$ under the stated assumptions and satisfies $0 \leq s_{j}(j=1, \ldots, m)$ and $\sum_{j=1}^{m} s_{j}=1$. For example, the signature of the $k$-out-of- $m$ system is defined by the condition $s_{m-k+1}=1$.

Received 28 November 2012; revision received 22 May 2013.

* Postal address: Department of Mathematics \& Statistics, McMaster University, 1280 Main Street West, Hamilton, Ontario L8S 4K1, Canada.

** Email address: bala@mcmaster.ca

*** Email address: volterwd@math.mcmaster.ca 
Given the system signature, the system reliability can be readily presented as

$$
\mathbb{P}(T>t)=\sum_{j=1}^{m} s_{j} \mathbb{P}\left(X_{j: m}>t\right)=\sum_{j=1}^{m} s_{j} \sum_{i=0}^{j-1}\left(\begin{array}{c}
m \\
i
\end{array}\right)[F(t)]^{i}[1-F(t)]^{m-i} .
$$

Much work has been done with regard to the properties of such representations and some others, and their applications. For a detailed review of all these developments, we refer the reader to the monograph of Samaniego [7].

Computation of signatures for complex systems can be quite intensive. Recently, Gertsbakh et al. [4] considered special cases in which the computational burden can be reduced. Specifically, they considered meta-systems wherein the modules are arranged into either series or parallel, and more generally recurrent systems. The signature for the meta-systems were easily obtained in these special cases.

Here, we consider a problem which arises in a different context and is a variation of the above scenario. That is, given a meta-system which failed due to its $i$ th subsystem or module, what is the conditional signature of the $i$ th module? We will refer to such a conditional signature vector as the ordered system signature. Such a representation could be useful for the analysis of meta-systems; see, for example, [9]. As done in [1], nonparametric inference may be developed based on the ordered system lifetimes.

The rest of this paper is organized as follows. In Section 2 we will formally define the ordered system signatures and establish some interesting properties. Then in Section 3 we will consider some special cases and illustrate the results developed here.

\section{The ordered system signature}

Let us consider a life-test where $n$ independent coherent systems are observed. We assume that the systems have $m$ components each and share a system signature $s$. The component lifetimes $X_{1}^{(k)}, \ldots, X_{m}^{(k)}, 1 \leq k \leq n$, are i.i.d. with a common continuous distribution function $F$. Let us denote the ordered component lifetimes of the $k$ th system by $X_{1: m}^{(k)}, \ldots, X_{m: m}^{(k)}$, and the corresponding system lifetime by $T_{k}$. Now, let the system lifetimes $T_{1}, \ldots, T_{n}$ be arranged in ascending order to give the ordered system lifetimes $T_{1: n}, \ldots, T_{n: n}$.

It is intuitive to think that when $s$ is nontrivial (i.e. not a $k$-out-of- $m$ system), early systems' failures are more likely to have failed due to critical components. More generally, this means that the signature vector of the $i$ th ordered system lifetime will change accordingly. The ordered system signature $\boldsymbol{s}^{(i: n)}=\left(s_{1}^{(i: n)}, \ldots, s_{m}^{(i: n)}\right)$ is thus defined as

$$
s_{j}^{(i: n)}=\sum_{k=1}^{n} \mathbb{P}\left(T_{i: n}=X_{j: m}^{(k)}\right), \quad j=1,2, \ldots, m,
$$

which is the probability the $i$ th module failure occurred to a module which failed due to the $j$ th ordered component failure. Under the assumption of i.i.d. continuous system lifetimes, we have the equivalent relation

$$
s_{j}^{(i: n)}=\mathbb{P}\left(T_{k}=X_{j: m}^{(k)} \mid T_{i: n}=T_{k}\right), \quad j=1,2, \ldots, m,
$$

for any $k=1,2, \ldots, n$.

Since the underlying system signature is free of the component lifetime distribution, it would be natural to expect a similar property to hold for the ordered system signature. This intuition is in fact confirmed in the following proposition. 
Proposition 1. The vector $\boldsymbol{s}^{(i: n)}=\left(s_{1}^{(i: n)}, \ldots, s_{m}^{(i: n)}\right)$ is free of the underlying component lifetime distribution $F$.

Proof. Let $\ell_{j}$ represent the number of modules which failed due to the $j$ th ordered component failure for $j=1,2, \ldots, m$. Under the assumption of the independence of modules, we have $\left(\ell_{1}, \ldots, \ell_{m}\right)$ to be distributed as multinomial with parameters $\left(n, s_{1}, \ldots, s_{m}\right)$. Let $\mathcal{L}=\left\{\ell=\left(\ell_{1}, \ldots, \ell_{m}\right): \ell_{1}+\cdots+\ell_{m}=n\right\}$. Then it follows that

$$
s_{j}^{(i: n)}=\sum_{\mathscr{L}}\left(\begin{array}{c}
n \\
\ell_{1}, \ldots, \ell_{m}
\end{array}\right)\left\{\prod_{k=1}^{m} s_{k}^{\ell_{k}}\right\} p_{j \mid \ell}^{(i: n)},
$$

where $p_{j \mid \ell}^{(i: n)}$ is the probability that the $i$ th module failed due to the $j$ th ordered component failure, given that $\ell_{k}$ modules failed due to the $k$ th ordered component failure for $k=1,2, \ldots, m$.

The event that the $i$ th failed module is due to the $j$ th ordered component failure, given $\ell$, can be decomposed into orderings of $X_{j_{k}: m}^{(k)}$. Such orderings of continuous and independent order statistics are distribution free, and, thus, so is $p_{j \mid \ell}^{(i: n)}$.

Remark 1. We can obtain $s_{j}^{(i: n)}$ by rephrasing the life-test in terms of a meta-system instead. Given a coherent meta-system with $n$ modules, having a common signature $s$, which failed due to the $i$ th module, what is the probability that this module failed due to its $j$ th ordered component failure? In this context $s_{j}^{(i: n)}$ can be viewed as a conditional probability under the assumption of independent modules with common system signature $\boldsymbol{s}$.

When viewing the ordered system lifetimes in terms of a conditional probability in a metasystem, if the modules are neither independent nor have a common signature $s$, then (1) will not hold. Furthermore, when the modules do not have common signature, the ordered system signature will in fact depend both on the structure of the system and the placement of the modules within the system.

It is clear that (1) provides a direct method for the computation of the ordered system signatures which can be expressed in two parts: one depending only on the common signature $s$, and the other involving a probability of orderings amongst order statistics which is free of $s$. Unfortunately, this also reveals that to obtain $s^{(i: n)}$ a large number of probabilities involving permutations of continuous and independent order statistics must be computed, even if only once. When $\mathrm{nm}$ is small, this is quite feasible, but when $\mathrm{nm}$ becomes large, the required computation becomes quite heavy. However, the number of ordering probabilities that need to be computed can be reduced by noting the following.

Lemma 1. The conditional probabilities $p_{j \mid \ell}^{(i: n)}$ satisfy

$$
\begin{gathered}
\ell_{j}=n \Longrightarrow p_{j \mid \ell}^{(i: n)}=1, \\
\ell_{j}=0 \Longrightarrow p_{j \mid \ell}^{(i: n)}=0, \\
\sum_{i=1}^{n} p_{j \mid \ell}^{(i: n)}=\ell_{j}, \\
p_{j \mid \ell}^{(i: n)}=p_{m-j+1 \mid \mathrm{rev} \ell}^{(n-i+1: n)}
\end{gathered}
$$

where rev $\ell=\left(\ell_{m}, \ldots, \ell_{1}\right)$ is simply the reverse ordering of $\ell=\left(\ell_{1}, \ldots, \ell_{m}\right)$.

Proof. Equations (2) and (3) are trivially true, whereas (4) follows as, conditioning on observing exactly $\ell_{j}$ systems failing due to the $j$ th ordered component failure, the total number 
over all ordered system lifetimes must be preserved. For (5), the left-hand side is the probability of a class of orderings of order statistics (OS) from distribution $F_{X}$. However, using the transformation $X \rightarrow-X$, the considered probability is equivalent to the probability on the right based on OS from $F_{-X}$. Since these probabilities are distribution-free, they must be identical.

To further reduce the necessary computations, we observe the following.

Corollary 1. For any signature vector $\boldsymbol{s}$ and all $n=1,2,3, \ldots, \boldsymbol{s}_{j}^{(i: n)}=0$ if and only if $s_{j}=0$.

Corollary 1 follows immediately from (1), (3), and the nonnegativity of the signature vector. With this, we need only compute $p_{j \mid \ell}^{(i: n)}$, where $\ell_{j}=0$ for all $j$ such that $s_{j}=0$.

An alternate representation to (1) is

$$
s_{j}^{(i: n)}=\sum_{\ell_{j}=1}^{n}\left(\begin{array}{l}
n \\
\ell_{j}
\end{array}\right) s_{j}^{\ell_{n}}\left(1-s_{j}\right)^{n-\ell_{j}} p_{j \mid \ell_{j}}^{(i: n)},
$$

where $p_{j \mid \ell_{j}}^{(i: n)}$ is the probability that the $i$ th module failed due to the $j$ th ordered component failure, given that exactly $\ell_{j}$ modules failed due to the $j$ th ordered component failure. Generally, $p_{j \mid \ell_{j}}^{(i: n)}$ will depend on the system signature $\boldsymbol{s}$.

The ordered system signature vectors satisfy some other interesting properties which can also lead to some simplification in the computation.

Proposition 2. The ordered system signatures satisfy

$$
\frac{1}{n} \sum_{i=1}^{n} s^{(i: n)}=s
$$

and

$$
\operatorname{rev} \boldsymbol{s}^{(i: n)}=(\operatorname{rev} \boldsymbol{s})^{(n-i+1: n)} .
$$

Proof. For the first part, we note that

$$
\sum_{i=1}^{n} s_{j}^{(i: n)}=\sum_{i=1}^{n} \sum_{\mathcal{L}}\left(\begin{array}{c}
n \\
\ell_{1}, \ldots, \ell_{m}
\end{array}\right)\left\{\prod_{k=1}^{m} s_{k}^{\ell_{k}}\right\} p_{j \mid \ell}^{(i: n)}=\sum_{\mathcal{L}}\left(\begin{array}{c}
n \\
\ell_{1}, \ldots, \ell_{m}
\end{array}\right)\left\{\prod_{k=1}^{m} s_{k}^{\ell_{k}}\right\} \ell_{j},
$$

which follows immediately from (4). The final expression can be viewed as an expectation of the $j$ th component of a multinomial random variable, and the required result then follows immediately.

For the second part, $\ell$ can be relabeled as rev $\ell$. Neither the multinomial coefficient nor the product $\prod s_{k}^{\ell_{k}}$ is changed. The result then follows from (5).

Corollary 2. If the system signature is symmetric (i.e. $s=$ rev $s$ ), then the ordered system signatures satisfy the following symmetry property:

$$
\operatorname{rev} \boldsymbol{s}^{(i: n)}=\boldsymbol{s}^{(n-i+1: n)} .
$$

The corollary follows immediately from Proposition 2.

Consider the partial ordering between signatures based on the same number of components $m$, as follows. We say that $s_{\mathrm{st}_{\mathrm{st}}}\left(\leq_{\mathrm{st}}\right) \boldsymbol{s}^{\prime}$ if $\sum_{j=1}^{k} s_{j} \leq(\geq) \sum_{j=1}^{k} s_{j}^{\prime}$ for all $k=1,2, \ldots, m$. See [5] where this ordering between signature vectors is used to imply ordering between the corresponding system lifetimes. Such orderings have also been used in other contexts, such as in comparing progressive censoring schemes [3]. 
Proposition 3. For any $1 \leq i_{1}<i_{2} \leq n$, the ordered system lifetimes satisfy $\boldsymbol{s}^{\left(i_{1}: n\right)} \leq_{\mathrm{st}} \boldsymbol{s}^{\left(i_{2}: n\right)}$. In addition, if $\boldsymbol{s}^{\left(i_{1}: n\right)} \geq_{\mathrm{st}} \boldsymbol{s}^{\left(\overline{i_{2}}: n\right)}$ for any $1 \leq i_{1}<i_{2} \leq n$ then $\boldsymbol{s}$ is the signature for a $k$-out-of- $m$ system.

Proof. For proving the first part, we only need to show that $\sum_{j=1}^{k} p_{j \mid \ell}^{\left(i_{1}: n\right)} \geq \sum_{j=1}^{k} p_{j \mid \ell}^{\left(i_{2}: n\right)}$, $k=1,2, \ldots, m$. Each sum can be seen as the probability that the $i$ th system failure had no more than $k$ components failed, conditional on $\ell$.

We say that $T_{i: n} \in L$ if the $i$ th system failed due to the failure of no more than $k$ components, and $T_{i: n} \in U$ otherwise. Furthermore, if $T_{i: n}$ fails due to the failure of exactly $j$ components, $1 \leq j \leq k$, we say that $T_{i: n}=L_{j}$, and if $k+1 \leq j \leq m$, then $T_{i: n}=U_{j}$. So $L=\bigcup_{j=1}^{k} L_{j}$ and $U=\bigcup_{k+1}^{m} U_{j}$, and it follows that

$$
\begin{aligned}
\sum_{j=1}^{k} p_{j \mid \ell}^{\left(i_{1}: n\right)} & =\mathbb{P}\left(T_{i_{1}: n} \in L \mid \ell\right) \\
& =\mathbb{P}\left(T_{i_{1}: n} \in L, T_{i_{2}: n} \in L \mid \ell\right)+\mathbb{P}\left(T_{i_{1}: n} \in L, T_{i_{2}: n} \in U \mid \ell\right) \\
& \geq \mathbb{P}\left(T_{i_{1}: n} \in L, T_{i_{2}: n} \in L \mid \ell\right)+\mathbb{P}\left(T_{i_{1}: n} \in U, T_{i_{2}: n} \in L \mid \ell\right) \\
& =\mathbb{P}\left(T_{i_{2}: n} \in L \mid \ell\right) \\
& =\sum_{j=1}^{k} p_{j \mid \ell}^{\left(i_{2}: n\right)} .
\end{aligned}
$$

To prove the inequality, we have

$$
\begin{aligned}
\mathbb{P}\left(T_{i_{1}: n} \in L, T_{i_{2}: n} \in U \mid \boldsymbol{\ell}\right) & =\sum_{j_{L}=1}^{k} \sum_{j_{U}=k+1}^{m} \mathbb{P}\left(T_{i_{1}: n}=L_{j_{L}}, T_{i_{2}: n}=U_{j_{U}} \mid \boldsymbol{\ell}\right) \\
& \geq \sum_{j_{L}=1}^{k} \sum_{j_{U}=k+1}^{m} \mathbb{P}\left(T_{i_{1}: n}=U_{j_{U}}, T_{i_{2}: n}=L_{j_{L}} \mid \boldsymbol{\ell}\right) \\
& =\mathbb{P}\left(T_{i_{1}: n} \in U, T_{i_{2}: n} \in L \mid \boldsymbol{\ell}\right) .
\end{aligned}
$$

The inequality follows by first conditioning on the $i_{1}$ th and $i_{2}$ th system failures failing at times $u$ and $v$, respectively, with $u<v$, and then unconditioning as

$$
\begin{aligned}
\mathbb{P}\left(T_{i_{1}: n}\right. & \left.=L_{j_{L}}, T_{i_{2}: n}=U_{j_{U}} \mid \ell\right) \\
& =\sum_{S \in \mathfrak{S}} \int_{0}^{1} \int_{0}^{v} C\left(S, u, v, j_{U}, j_{L}\right) u^{j_{L}-1}(1-u)^{m-j_{L}} v^{j_{U}-1}(1-v)^{m-j_{U}} \mathrm{~d} u \mathrm{~d} v \\
& \geq \sum_{S \in \mathfrak{S}} \int_{0}^{1} \int_{0}^{v} C\left(S, u, v, j_{U}, j_{L}\right) u^{j_{U}-1}(1-u)^{m-j_{U}} v^{j_{L}-1}(1-v)^{m-j_{L}} \mathrm{~d} u \mathrm{~d} v \\
& =\mathbb{P}\left(T_{i_{1}: n}=U_{j_{U}}, T_{i_{2}: n}=L_{j_{L}} \mid \ell\right) .
\end{aligned}
$$

Here $\mathfrak{S}$ represents all possible placements $S$ of the $n-2$ remaining system lifetimes such that $\left(i_{1}-1\right),\left(i_{2}-i_{1}-1\right)$, and $\left(n-i_{1}-i_{2}\right)$ system lifetimes fall in $(0, u),(u, v)$, and $(v, 1)$, respectively. Then $C\left(S, u, v, j_{U}, j_{L}\right)$ is simply the product of the multinomial probabilities representing the probability of $S$, and the normalizing constants for the appropriate beta distributions. This constant is positive, and, for all $0<u<v<1$, we have

$$
u^{a-1}(1-u)^{m-a} v^{b-1}(1-v)^{m-b}>u^{b-1}(1-u)^{m-b} v^{a-1}(1-v)^{m-a}
$$


whenever $a<b$. Setting $a=j_{L}$ and $b=j_{U}$ yields the inequality. Equality holds if and only if $\mathbb{P}\left(T_{i_{1}: n}=L_{j_{L}}, T_{i_{2}: n}=U_{j_{U}} \mid \ell\right)=0$, in which case the set $\mathfrak{S}$ is empty.

To prove the second part, we have, for some $i_{1} \neq i_{2}, s^{\left(i_{2}: n\right)} \geq_{\mathrm{st}} s^{\left(i_{1}: n\right)}$ and $s^{\left(i_{1}: n\right)} \geq_{\mathrm{st}} s^{\left(i_{2}: n\right)}$, which immediately implies that $s^{\left(i_{1}: n\right)}=s^{\left(i_{2}: n\right)}$. Let $\kappa$ be the smallest $k$ such that $s_{k}>0$. If only one such $k$ exists, we are done. Let $\kappa^{\prime}$ be any other $k$ such that $s_{k}>0$, and so $\kappa<\kappa^{\prime}$ and

$$
0=\sum_{j=1}^{\kappa}\left(s_{j}^{\left(i_{1}: n\right)}-s_{j}^{\left(i_{2}: n\right)}\right)=\sum_{\mathcal{L}}\left(\begin{array}{c}
n \\
\ell_{1}, \ldots, \ell_{m}
\end{array}\right)\left\{\prod_{k=1}^{m} s_{j}^{\ell_{k}}\right\} \sum_{j=1}^{\kappa}\left[p_{j \mid \ell}^{\left(i_{1}: n\right)}-p_{j \mid \ell}^{\left(i_{2}: n\right)}\right] .
$$

Let $\ell$ be such that $\ell_{\kappa}=1$ and $\ell_{\kappa^{\prime}}=n-1>0$. Then

$$
\sum_{j=1}^{\kappa}\left[p_{j \mid \ell}^{\left(i_{1}: n\right)}-p_{j \mid \ell}^{\left(i_{2}: n\right)}\right]=p_{\kappa \mid \ell}^{\left(i_{1}: n\right)}-p_{\kappa \mid \ell}^{\left(i_{2}: n\right)}>0
$$

as $\mathbb{P}\left(T_{i_{1}: n}=L_{\kappa}, T_{i_{2}: n}=U_{\kappa^{\prime}} \mid \ell\right)>0$, since both $\ell_{\kappa}$ and $\ell_{\kappa^{\prime}}$ are nonzero. Consequently, the right-hand side of (7) is 0 only if $s_{\kappa} s_{\kappa^{\prime}}^{n-1}=0$, which contradicts the existence of $\kappa^{\prime}$.

The reverse statement for the second part of Proposition 3 is trivially true as shown in the subsequent section.

\section{Special cases and computation}

Closed-form solutions for the ordered system signatures are in general not available. However, there are two situations in which the ordered system signatures can be explicitly obtained. The simplest such scenario is the $k$-out-of- $m$ system. Equations (1) and (6) both simplify trivially, so that $\boldsymbol{s}^{(i: n)}=\boldsymbol{s}$ for $i=1,2, \ldots, n$ in this case.

A more interesting case that can be handled is the uniform signature, that is, when $s=$ $(1 / m, \ldots, 1 / m)$ is a signature vector of size $m$. In such a case, the system lifetime $T$ has the same distribution as the individual component lifetimes. Using this fact, a combinatorial argument can then be used to show that, for $j=1,2, \ldots, m$ and $i=1,2, \ldots, n$, we have

$$
s_{j}^{(i: n)}=\frac{\left(\begin{array}{c}
i+j-2 \\
j-1
\end{array}\right)\left(\begin{array}{c}
m+n-i-j \\
m-j
\end{array}\right)}{\left(\begin{array}{c}
m+n-1 \\
n
\end{array}\right)} .
$$

While $s=(1 / m, \ldots, 1 / m)$ is not the signature vector of a coherent system itself and is in fact the signature of a mixed system, (8) can be used to either further reduce the computation of the conditional probabilities $p_{j \mid \ell}^{(i: n)}$, or for the purpose of verifying the computations. See [2] for a discussion on mixed systems.

\subsection{Examples}

We consider the computation of the ordered system signatures for some specific cases of $m$ and $n$ to illustrate the results established in the preceding section. In Tables $1-4$ we present the conditional probabilities $p_{j \mid \ell}^{(i: n)}$ necessary to compute all ordered system signatures with three and four components for sample sizes 2 and 3. The required computations were reduced using Lemma 1, so that for Tables 1, 2, 3, and 4 only 2, 8, 4, and 20 conditional probabilities $p_{j \mid \ell}^{(i: n)}$ were explicitly computed, respectively. The uniform ordered system signature in (8) was used to verify the correctness of the computations.

The ordered system signatures for all coherent systems with three or four components are presented in Table 5, which have been computed from (1) with the conditional probabilities 
TABle 1: Conditional probabilities $p_{j \mid \ell}^{(i: n)}$ for $i=1,2, \ldots, n, j=1,2, \ldots, m, m=3$, and $n=2$.

\begin{tabular}{ccccc}
\hline & & \multicolumn{3}{c}{$\left(\ell_{1}, \ell_{2}, \ell_{3}\right)$} \\
\cline { 3 - 5 } & & $(1,1,0)$ & $(1,0,1)$ & $(0,1,1)$ \\
\hline 1 & 1 & $\frac{4}{5}$ & $\frac{19}{20}$ & 0 \\
& 2 & $\frac{1}{5}$ & 0 & $\frac{4}{5}$ \\
& 3 & 0 & $\frac{1}{20}$ & $\frac{1}{5}$ \\
\hline 2 & 1 & $\frac{1}{5}$ & $\frac{1}{20}$ & 0 \\
& 3 & $\frac{4}{5}$ & 0 & $\frac{1}{5}$ \\
& 2 & 0 & $\frac{19}{20}$ & $\frac{4}{5}$ \\
\hline
\end{tabular}

TABLE 2: Conditional probabilities $p_{j \mid \ell}^{(i: n)}$ for $i=1,2, \ldots, n, j=1,2, \ldots, m, m=3$, and $n=3$.

\begin{tabular}{ccccccccc}
\hline & $i$ & $j$ & \multicolumn{7}{c}{$\left(\ell_{1}, \ell_{2}, \ell_{3}\right)$} \\
\cline { 2 - 8 } & & $(2,1,0)$ & $(2,0,1)$ & $(1,2,0)$ & $(0,2,1)$ & $(1,0,2)$ & $(0,1,2)$ & $(1,1,1)$ \\
\hline 1 & 1 & $\frac{11}{12}$ & $\frac{83}{84}$ & $\frac{29}{42}$ & 0 & $\frac{383}{420}$ & 0 & $\frac{131}{168}$ \\
& 2 & $\frac{1}{12}$ & 0 & $\frac{13}{42}$ & $\frac{191}{210}$ & 0 & $\frac{287}{420}$ & $\frac{32}{168}$ \\
& 3 & 0 & $\frac{1}{84}$ & 0 & $\frac{19}{210}$ & $\frac{37}{420}$ & $\frac{133}{420}$ & $\frac{5}{168}$ \\
\hline 2 & 1 & $\frac{23}{30}$ & $\frac{97}{105}$ & $\frac{23}{105}$ & 0 & $\frac{8}{105}$ & 0 & $\frac{32}{168}$ \\
& 2 & $\frac{7}{30}$ & 0 & $\frac{82}{105}$ & $\frac{82}{105}$ & 0 & $\frac{7}{30}$ & $\frac{104}{168}$ \\
& 3 & 0 & $\frac{8}{105}$ & 0 & $\frac{23}{105}$ & $\frac{97}{105}$ & $\frac{23}{30}$ & $\frac{32}{168}$ \\
\hline 3 & 1 & $\frac{133}{420}$ & $\frac{37}{420}$ & $\frac{19}{210}$ & 0 & $\frac{1}{84}$ & 0 & $\frac{5}{168}$ \\
& 2 & $\frac{287}{420}$ & 0 & $\frac{191}{210}$ & $\frac{13}{42}$ & 0 & $\frac{1}{12}$ & $\frac{32}{168}$ \\
& 3 & 0 & $\frac{383}{420}$ & 0 & $\frac{29}{42}$ & $\frac{83}{84}$ & $\frac{11}{12}$ & $\frac{131}{168}$ \\
\hline
\end{tabular}

TABLE 3: Conditional probabilities $p_{j \mid \ell}^{(i: n)}$ for $i=1,2, \ldots, n, j=1,2, \ldots, m, m=4$, and $n=2$.

\begin{tabular}{cccccccc}
\hline & & \multicolumn{6}{c}{$\left(\ell_{1}, \ell_{2}, \ell_{3}, \ell_{4}\right)$} \\
\cline { 2 - 7 } & & $(1,1,0,0)$ & $(1,0,1,0)$ & $(1,0,0,1)$ & $(0,1,1,0)$ & $(0,1,0,1)$ & $(0,0,1,1)$ \\
\hline \multirow{2}{*}{1} & 1 & $\frac{11}{14}$ & $\frac{13}{14}$ & $\frac{69}{70}$ & 0 & 0 & 0 \\
& 2 & $\frac{3}{14}$ & 0 & 0 & $\frac{53}{70}$ & $\frac{13}{14}$ & 0 \\
& 3 & 0 & $\frac{1}{14}$ & 0 & $\frac{17}{70}$ & 0 & $\frac{11}{14}$ \\
& 4 & 0 & 0 & $\frac{1}{70}$ & 0 & $\frac{1}{14}$ & $\frac{3}{14}$ \\
\hline 2 & 1 & $\frac{3}{14}$ & $\frac{1}{14}$ & $\frac{1}{70}$ & 0 & 0 & 0 \\
& 2 & $\frac{11}{14}$ & 0 & 0 & $\frac{17}{70}$ & $\frac{1}{14}$ & 0 \\
& 3 & 0 & $\frac{13}{14}$ & 0 & $\frac{53}{70}$ & 0 & $\frac{3}{14}$ \\
& 3 & 0 & 0 & $\frac{69}{70}$ & 0 & $\frac{13}{14}$ & $\frac{11}{14}$ \\
\hline
\end{tabular}




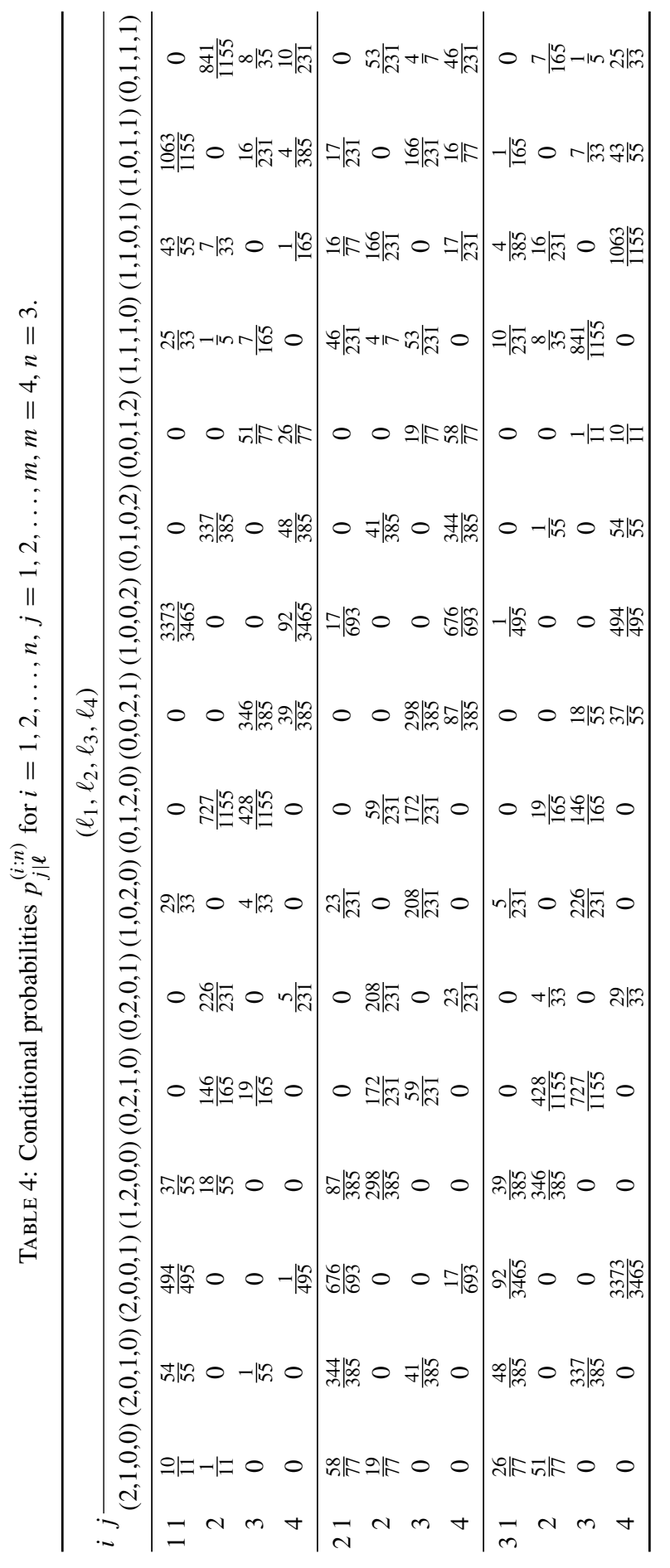


TABLE 5: List of ordered system signatures for all system signatures $s$ of coherent systems of size 3 and 4 , omitting $k$-out-of- $m$ systems and systems with reverse signatures.

\begin{tabular}{|c|c|c|}
\hline \multicolumn{3}{|c|}{$m=3$ and $n=2,3$} \\
\hline$s=\frac{1}{3}(1,2,0)$ & & \\
\hline $\boldsymbol{s}^{(1: 2)}=\frac{1}{15}(7,8,0)$ & $\boldsymbol{s}^{(2: 2)}=\frac{1}{15}(3,12,0)$ & \\
\hline $\boldsymbol{s}^{(1: 3)}=\frac{1}{210}(115,95,0)$ & $\boldsymbol{s}^{(2: 3)}=\frac{1}{210}(64,146,0)$ & $\boldsymbol{s}^{(3: 3)}=\frac{1}{210}(31,179,0)$ \\
\hline \multicolumn{3}{|c|}{$m=4$ and $n=2,3$} \\
\hline \multicolumn{3}{|l|}{$s=\frac{1}{2}(1,1,0,0)$} \\
\hline $\boldsymbol{s}^{(1: 2)}=\frac{1}{14}(9,5,0,0)$ & $s^{(2: 2)}=\frac{1}{14}(5,9,0,0)$ & \\
\hline $\boldsymbol{s}^{(1: 3)}=\frac{1}{770}(553,217,0,0)$ & $s^{(2: 3)}=\frac{1}{770}(379,391,0,0)$ & $\boldsymbol{s}^{(3: 3)}=\frac{1}{770}(223,547,0,0)$ \\
\hline \multicolumn{3}{|l|}{$s=\frac{1}{4}(1,3,0,0)$} \\
\hline $\boldsymbol{s}^{(1: 2)}=\frac{1}{14}(5,9,0,0)$ & $\boldsymbol{s}^{(2: 2)}=\frac{1}{14}(2,12,0,0)$ & \\
\hline $\boldsymbol{s}^{(1: 3)}=\frac{1}{1540}(658,882,0,0)$ & $\boldsymbol{s}^{(2: 3)}=\frac{1}{1540}(334,1206,0,0)$ & $\boldsymbol{s}^{(3: 3)}=\frac{1}{1540}(163,1377,0,0)$ \\
\hline \multicolumn{3}{|l|}{$s=\frac{1}{12}(3,7,2,0)$} \\
\hline $\boldsymbol{s}^{(1: 2)}=\frac{1}{420}(155,231,34,0)$ & \multicolumn{2}{|l|}{$s^{(2: 2)}=\frac{1}{420}(55,259,106,0)$} \\
\hline \multicolumn{3}{|c|}{$\boldsymbol{s}^{(1: 3)}=\frac{1}{9240}(4123,4641,476,0) \boldsymbol{s}^{(2: 3)}=\frac{1}{9240}(1984,5964,1292,0) \boldsymbol{s}^{(3: 3)}=\frac{1}{9240}(823,5565,2852,0)$} \\
\hline \multicolumn{3}{|l|}{$s=\frac{1}{4}(1,1,2,0)$} \\
\hline $\boldsymbol{s}^{(1: 2)}=\frac{1}{140}(55,39,46,0)$ & $\boldsymbol{s}^{(2: 2)}=\frac{1}{140}(15,31,94,0)$ & \\
\hline $\boldsymbol{s}^{(1: 3)}=\frac{1}{3080}(1505,843,732,0)$ & $s^{(2: 3)}=\frac{1}{3080}(620,888,1572,0)$ & $\boldsymbol{s}^{(3: 3)}=\frac{1}{3080}(185,579,2316,0)$ \\
\hline \multicolumn{3}{|l|}{$s=\frac{1}{6}(0,5,1,0)$} \\
\hline$s^{(1: 2)}=\frac{1}{21}(0,19,2,0)$ & $s^{(2: 2)}=\frac{1}{21}(0,16,5,0)$ & \\
\hline $\boldsymbol{s}^{(1: 3)}=\frac{1}{924}(0,859,65,0)$ & $\boldsymbol{s}^{(2: 3)}=\frac{1}{924}(790,134)$ & $\boldsymbol{s}^{(3: 3)}=\frac{1}{924}(0,661,263,0)$ \\
\hline \multicolumn{3}{|l|}{$s=\frac{1}{3}(0,2,1,0)$} \\
\hline $\boldsymbol{s}^{(1: 2)}=\frac{1}{105}(0,82,23,0)$ & $s^{(2: 2)}=\frac{1}{105}(0,58,47,0)$ & \\
\hline $\boldsymbol{s}^{(1: 3)}=\frac{1}{1155}(0,958,197,0)$ & $s^{(2: 3)}=\frac{1}{1155}(0,790,365,0)$ & $s^{(3: 3)}=\frac{1}{1155}(0,562,593,0)$ \\
\hline \multicolumn{3}{|l|}{$s=\frac{1}{2}(0,1,1,0)$} \\
\hline $\boldsymbol{s}^{(1: 2)}=\frac{1}{35}(0,22,13,0)$ & $s^{(2: 2)}=\frac{1}{35}(0,13,22,0)$ & \\
\hline $\boldsymbol{s}^{(1: 3)}=\frac{1}{140}(0,97,43)$, & $s^{(2: 3)}=\frac{1}{140}(0,70,70,0)$ & $s^{(3: 3)}=\frac{1}{140}(0,43,97,0)$ \\
\hline
\end{tabular}

computed in Tables 1-4. The reader is referred to [8] for the computation of the signatures of all coherent systems with three or four components. The $k$-out-of- $m$ systems and the systems with reverse ordered system signatures have been omitted from Table 5.

It should be noted that, for coherent systems, it is not necessary to obtain all conditional probabilities $p_{j \mid \ell}^{(i: n)}$. For example, when $\ell=(1,0,1)$, the probability of observing such an $\ell$ is 0 . However, for mixed systems with arbitrary signatures, it would be necessary to obtain all conditional probabilities $p_{j \mid \ell}^{(i: n)}$.

\section{References}

[1] Balakrishnan, N., NG, H. K. T. and Navarro, J. (2011). Exact nonparametric inference for component lifetime distribution based on lifetime data from systems with known signatures. J. Nonparametric Statist. 23, 741-752. 
[2] Boland, P. J. and Samaniego, F. (2004). The signature of a coherent system and its applications in reliability. In Mathematical Reliability: An Expository Perspective. eds R. Soyer, T. Mazzuchi, and N. D. Singpurwalla, Kluwer, Boston, MA, pp. 3-30.

[3] Cramer, E. (2002). A note on moments of progressively type II censored order statistics. Commun. Statist. Theory Meth. 31, 1301-1307.

[4] Gertsbakh, I., Shpungin, Y. and Spizzichino, F. (2011). Signatures of coherent systems built with separate modules. J. Appl. Prob. 48, 843-855.

[5] Kochar, S., MukerJee, H. and Samaniego, F. J. (1999). The 'signature' of a coherent system and its application to comparisons among systems. Naval Res. Logistics 46, 507-523.

[6] Samaniego, F. J. (1985). On closure of the IFR class under formation of coherent systems. IEEE Trans. Reliab. R-34, 69-72.

[7] Samaniego, F. J. (2007). System Signatures and Their Applications in Engineering Reliability (Internat. Ser. Operat. Res. Management Sci. 110). Springer, New York.

[8] Shaked, M. and Suarez-Llorens, A. (2003). On the comparison of reliability experiments based on the convolution order. J. Amer. Statist. Assoc. 98, 693-702.

[9] Spizzichino, F. and Navarro, J. (2012). Signatures and symmetry properties of coherent systems. In Recent Advances in System Reliability, eds A. Lisnianski and I. Frenkel, Springer, London, pp. 33-48. 